NIST Technical Note 1944

\title{
OpenFOAM Large-Eddy Simulations of Atmospheric Boundary Layer Turbulence for Wind Engineering Applications
}

Liang Shi

DongHun Yeo

This publication is available free of charge from: https://doi.org/10.6028/NIST.TN.1944
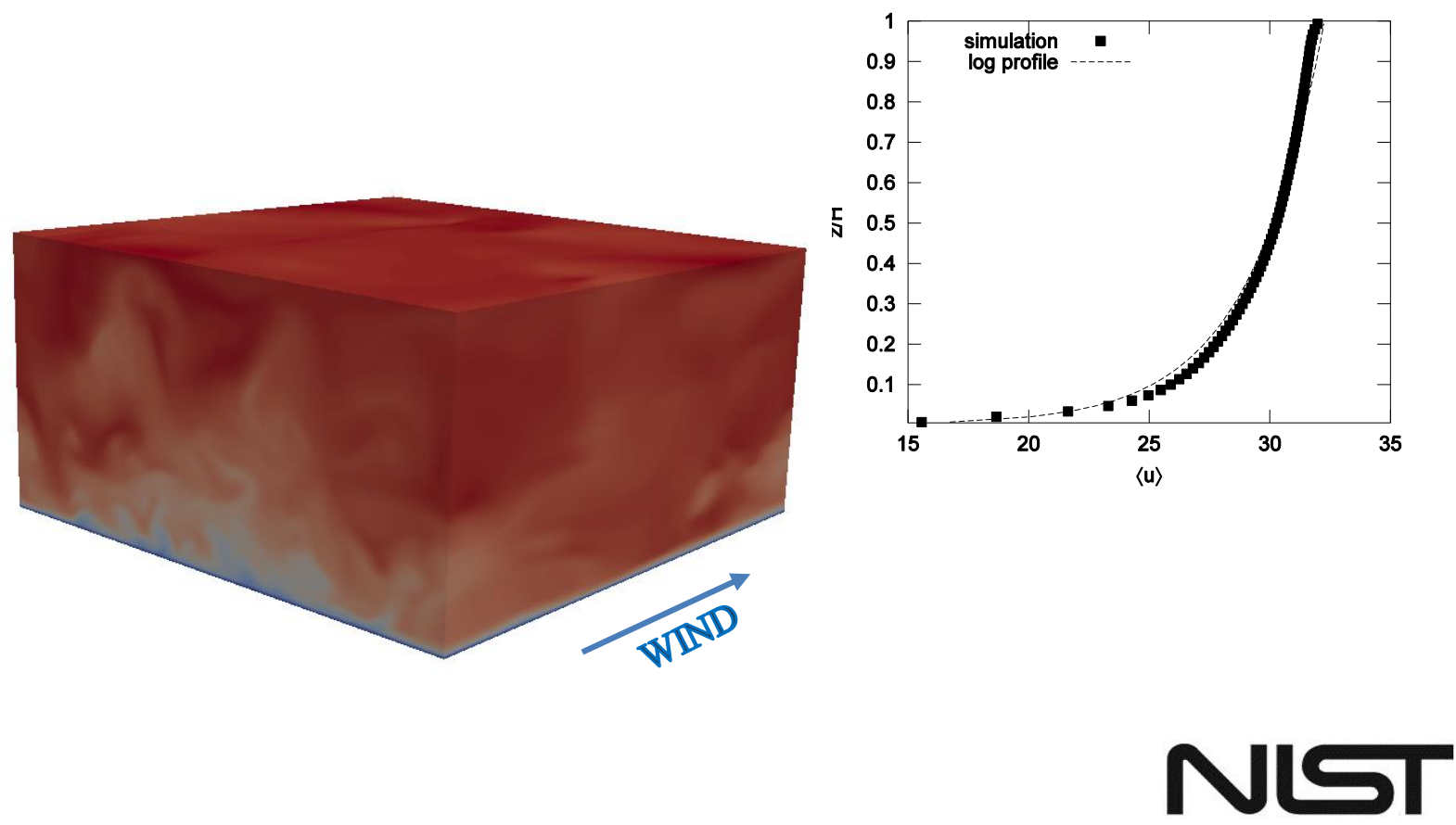

National Institute of Standards and Technology U.S. Department of Commerce 
NIST Technical Note 1944

\section{OpenFOAM Large-Eddy Simulations of Atmospheric Boundary Layer Turbulence for Wind Engineering Applications}

Liang Shi

DongHun Yeo

Engineering Laboratory

This publication is available free of charge from:

https://doi.org/10.6028/NIST.TN.1944

November 2016

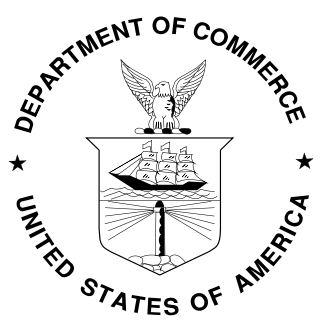

U.S. Department of Commerce

Penny Pritzker, Secretary

National Institute of Standards and Technology

Willie May, Under Secretary of Commerce for Standards and Technology and Director 
Certain commercial entities, equipment, or materials may be identified in this document in order to describe an experimental procedure or concept adequately. Such identification is not intended to imply recommendation or endorsement by the National Institute of Standards and Technology, nor is it intended to imply that the entities, materials, or equipment are necessarily the best available for the purpose.

National Institute of Standards and Technology Technical Note 1944 Natl. Inst. Stand. Technol. Tech. Note 1944, 32 pages (November 2016) CODEN: NTNOEF

This publication is available free of charge from: https://doi.org/10.6028/NIST.TN.1944 


\section{DISCLAIMER}

(1) The policy of the NIST is to use the International System of Units in its technical communications. In this document, however, works of authors outside NIST are cited which describe measurements in certain non-SI units. Thus, it is more practical to include the non-SI unit measurements from these references.

(2) Certain trade names or company products or procedures may be mentioned in the text to specify adequately the experimental procedure or equipment used. In no case does such identification imply recommendation or endorsement by the National Institute of Standards and Technology, nor does it imply that the products or procedures are the best available for the purpose. 


\begin{abstract}
Statistical properties such as the spectral density and spatial coherence of boundary layer turbulence affect bluff body aerodynamics and structural responses. In this report, the opensource toolbox OpenFOAM is employed to perform LES simulations of boundary layer flows with rough ground and to obtain turbulence statistics. The one-k-equation-eddy SGS model is used for the subgrid-scale motions while the wall shear model is applied at the ground. The mean velocity profiles follow the logarithmic law except the near-ground region owing to the limited accuracy of the SGS model. The Reynolds stresses, the third-order moments and the energy budgets are reasonably well represented. The power spectra agree with the modified Kaimal expressions at low frequencies. Additional research is planned on the simulation of higher frequency turbulence spectra. The spatial coherence functions are exponential and consistent with the expressions commonly used in wind engineering applications.
\end{abstract}

Keywords: Atmospheric boundary layer; large-eddy simulations (LES); OpenFOAM; turbulence; wind engineering 


\section{ACKNOWLEDGEMENT}

The authors wish to thank Dr. Emil Simiu of the National Institute of Standards and Technology for his insightful discussions and helpful comments on the draft of this report. Special thanks is given to Mr. Paul Dickey at the group of Engineering Laboratory System Administration (ELSA) for his excellent technical support. Dr. Liang Shi served as a NIST Director's Postdoctoral Researcher. 


\section{Contents}

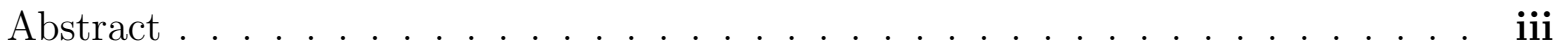

Acknowledgement ...................... . . iv

List of Figures . . . . . . . . . . . . . . . . . . . . . . vi

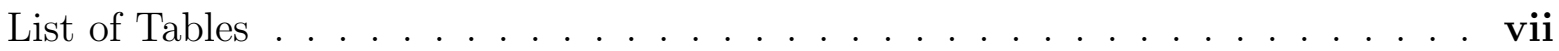

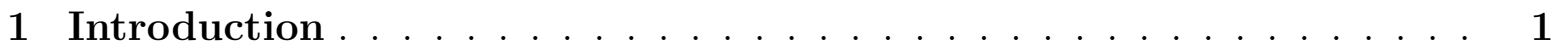

2 Mathematical formulation . . . . . . . . . . . . . . . . 3

2.1 Mean flow . . . . . . . . . . . . . . . . . . . . . 4

2.2 Energy budgets . . . . . . . . . . . . . . . . . . . . . . . . . . . . . 4

2.3 Subgrid-scale models . . . . . . . . . . . . . . . . . . . 6

3 Simulation specifications . . . . . . . . . . . . . . . . 7

3.1 Geometry, boundary and initial conditions . . . . . . . . . . . . . 7

3.2 OpenFOAM: a finite-volume numerical solver . . . . . . . . . . . . . 9

4 Horizontally-averaged flow statistics . . . . . . . . . . . . . . . 10

4.1 Mean velocity . . . . . . . . . . . . . . . . . . . . . . . . . . . . . . . . . .

4.2 Velocity variances . . . . . . . . . . . . . . . . . . . . . . . . . . . . . . . . . . . . . . . . .

4.3 Reynolds stresses . . . . . . . . . . . . . . . . . . . . . . 12

4.4 Fluxes of variances and Reynolds stresses . . . . . . . . . . . . . . . . 13

4.5 SGS kinetic energy, SGS viscosity, and SGS stress . . . . . . . . . . . 13

4.6 Turbulent kinetic energy budget . . . . . . . . . . . . . . . . . . 15

5 Probability density function, autocovariance, spectra, and spatial

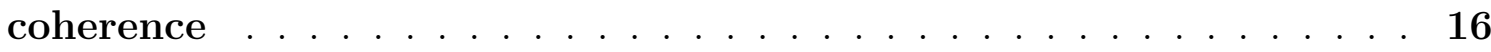

6 Conclusions ............................. 19

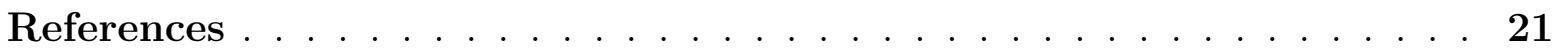




\section{List of Figures}

3.1 Computational geometry . . . . . . . . . . . . . . . . . . 8

4.1 Contours of filtered velocity fluctuactions at different heights . . . . . 10

4.2 Profiles of mean velocity and its gradient . . . . . . . . . . . . . . 11

4.3 Profiles of velocity variance and turbulent kinetic energy . . . . . . . . . 12

4.4 Profiles of Reynolds and SGS stresses . . . . . . . . . . . . . . . 13

4.5 Profiles of turbulent fluxes of variances and Reynolds stresses . . . . . . . 14

4.6 Profiles of SGS kinetic energy, SGS viscosity, and SGS stresses . . . . . . 14

4.7 Profiles of turbulent kinetic energy budget . . . . . . . . . . . . 15

5.1 Time series and PDF of 1-point velocity . . . . . . . . . . . . 16

5.2 Autocorrelation and spectral density function $\ldots \ldots \ldots \ldots$

5.3 Coherence function in $x$ direction $\ldots \ldots \ldots \ldots \ldots$

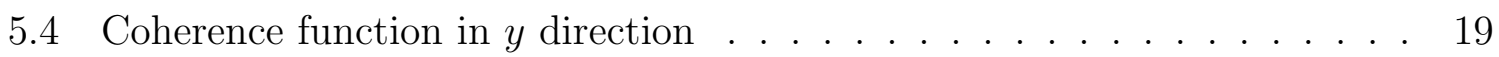

5.5 Coherence function in $z$ direction $\ldots \ldots \ldots \ldots \ldots$ 


\section{List of Tables}

3.1 Parameters in the simulations. . . . . . . . . . . . . . . . . . . . 7 


\section{Introduction}

Large-eddy simulation (LES) has been studied and developed since the groundbreaking work in the mid 1960s by Smagorinsky [37] and Lilly [21]. Significant theoretical progresses on LES of wall-bounded flows have been made, from subgrid-scale (SGS) modeling [19, 22], wall-layer modelling [28] to numerical issues [16]. Deardorff [11] introduced a variant of Smagorinsky model by solving a transport equation for SGS turbulent kinetic energy. Schumann [34] developed a two-part eddy viscosity model that takes into account of the mean shear near the ground and a wall stress model that is still widely used. The Germano identity [12] uses the assumption of scale invariance near the cut-off scale and was the basis of the dynamic Smagorinsky model. Moin et al. $[16,25]$ conducted a landmark study on the effects of the numerical errors in LES of turbulence. Porte-Agel and Meneveau [31] developed a scale-dependent SGS model which removes the assumption of scale invariance in the dynamical Smagorinsky model and hence is more generally applicable. Most recently, a novel potential LES methodology of wall-bounded flow was introduced by Chen et al. [7], who applied the averaged momentum equilibrium constraint on the SGS model in the near wall region.

However, LES technique still faces numerous challenges in both theory [30] and practical applications [17]. First, in spite of the improvements being reported in many recent studies [15, 40], fundamental problems related to the SGS and wall modeling persist, especially for non-equilibrium boundary layer flow. Second, large-scale engineering applications of LES are still not feasible. One reason is that LES is computationally costly, even though computing speed is growing into the era of exascale flops; Another reason is that the fundamental research on LES is not closely connected to engineering. In-house LES code development remains a norm in the community. To reduce the gap between theoretical work and practical applications of LES, LES scientists and engineers need a common platform for research, communication, and innovation.

As an open source CFD (Computational Fluid Dynamics) toolbox written in objectoriented language $\mathrm{C}++$, OpenFOAM (Open Field Operation And Manipulation) has been rapidly gaining prominence during the last decade [26, 6]. Applications of OpenFOAM range from classical turbulence simulation to multiphysics problems including heat and electromagnetic fields, and to financial mathematical models. Churchfield et al. [8] applied OpenFOAM in the wind engineering, where the atmospheric boundary layer is simulated with the original Smagorinsky model. Lignarolo et al. [20] investigated numerical issues in LES of boundary layer flows with OpenFOAM. Nevertheless, most of the research on LES are still based on in-house codes with relatively short lifetime and small user groups.

In this report, we employ OpenFOAM to develop a capability for adequately simulating flows of the atmospheric boundary layer type for future use in simulation of wind effects on structures. The open-source nature and the flexibility of OpenFOAM, together with other advantages [6], makes it a suitable choice for the purpose. In particular, we are interested in examining such properties of the turbulent flow as the spectral density and the spatial coherence, which affect bluff body aerodynamics and structural responses. The spatial coherence functions of atmospheric boundary layer flows have been rarely examined by CFD practitioners, and are specifically addressed in this work. 
The report is structured as follows. We provide a summary mathematical description of LES in Section 2. Simulation details are described in Section 3. The turbulence statistics are presented in Section 4 and 5. 


\section{Mathematical formulation}

The partial differential equations governing the motion of Newtonian incompressible flows are

$$
\begin{aligned}
\nabla \cdot \mathbf{u} & =0 \\
\partial_{t} \mathbf{u}+\mathbf{u} \nabla \mathbf{u} & =-\nabla p+\nu \nabla^{2} \mathbf{u}+F
\end{aligned}
$$

where $\mathbf{u}=(u, v, w)$ is the velocity field, $p$ is the reduced pressure $p_{h} / \rho, p_{h}$ is the dynamic pressure, $\rho$ is the fluid density, $\nu$ is the kinematic viscosity and $F$ is the external force. Equations (2.1a) and (2.1b) express, respectively, the incompressibility condition and momentum conservation. Coriolis and thermal stratification effects are not considered in Eqs. (2.1).

The LES equations are obtained by applying, to Eq. (2.1), a spatial low-pass filter, denoted by an overtilde:

$$
\begin{aligned}
\widetilde{\nabla \cdot \mathbf{u}} & =0, \\
\partial_{t} \widetilde{\mathbf{u}}+\widetilde{\mathbf{u} \nabla \mathbf{u}} & =-\nabla \widetilde{p}+\nu \nabla^{2} \widetilde{\mathbf{u}}+\widetilde{F} .
\end{aligned}
$$

The filtered quantity is defined as

$$
\widetilde{f}(\mathbf{r})=\int f\left(\mathbf{r}^{\prime}\right) G\left(\mathbf{r}, \mathbf{r}^{\prime}\right) d \mathbf{r}^{\prime}
$$

where $\mathbf{r}$ is the position vector, $\mathbf{r}^{\prime}$ is the variable of integration, $f$ is the integrand, and $G$ is the filter convolution kernel. Among numerous filters with different properties [29], a common one for the finite volume method is the top-hat filter, which physically means the volume average of a physical quantity on a cell. Hence, the finite volume method (e.g., in OpenFOAM) usually requires no explicit filtering operations to perform LES. Note that the operations of filtering and differentiation with respect to the spatial coordinates do not generally commute but do so for homogeneous filters, that is, filters not depending on the spatial coordinates. For homogeneous filters the incompressibility condition still holds, $\nabla \cdot \widetilde{\mathbf{u}}=0$, which yields

$$
\widetilde{\mathbf{u} \nabla \mathbf{u}}=\widetilde{\mathbf{u}} \nabla \widetilde{\mathbf{u}}+\nabla \cdot \tau
$$

where $\tau_{i j}=\widetilde{u_{i} u_{j}}-\widetilde{u_{i}} \widetilde{u_{j}}$ is called the residual stress tensor or subgrid-scale (SGS) stress tensor. Since the isotropic component of the SGS stress can be integrated into the pressure term, the deviatoric component $\tau_{i j}^{d}=\tau_{i j}-\frac{1}{3} \delta_{i j} \tau_{k k}$ must be modeled using the filtered quantities $\widetilde{\mathbf{u}}$ and $\widetilde{p}$. For homogeneous filters, Equations (2.2) can be reorganized as

$$
\begin{aligned}
\nabla \cdot \widetilde{\mathbf{u}} & =0, \\
\partial_{t} \widetilde{\mathbf{u}}+\widetilde{\mathbf{u}} \nabla \widetilde{\mathbf{u}} & =-\nabla \widetilde{p}+\nu \Delta \widetilde{\mathbf{u}}-\nabla \cdot \tau^{d}+\widetilde{F} .
\end{aligned}
$$

In this report, Equations 2.5 are numerically integrated for boundary layer flows. 


\subsection{Mean flow}

Next we derive the equations for mean-flow velocity, mean-flow kinetic energy, total kinetic energy, and turbulent kinetic energy of the resolved flow field. Since we are interested in statistically stationary turbulence in boundary layer flows, mean-flow quantities are defined in this report as the statistical average in the homogeneous directions, denoted by an angle bracket,

$$
\langle f(z)\rangle=\frac{1}{T} \frac{1}{L_{x}} \frac{1}{L_{y}} \quad f(t ; x, y, z) d y d x d t .
$$

$T$ is the total time, and $L_{x}, L_{y}$ are the domain length in $x$ and $y$ directions. The $x$ and $y$ directions are along-wind and cross-wind direction, respectively. In the vertical $z$ direction, the statistics of physical quantities vary with the distance from the ground. By applying this averaging operator to Eqs. (2.5), we obtain the mean-flow equations (for simplicity overtilde for single variable is henceforth omitted)

$$
\begin{aligned}
\frac{d\langle w\rangle}{d z} & =0, \\
\frac{d}{d z}\left(\langle u\rangle\langle w\rangle+\left\langle u^{\prime} w^{\prime}\right\rangle+\left\langle\tau_{x z}\right\rangle-\nu \frac{d\langle u\rangle}{d z}\right) & =F, \\
\frac{d}{d z}\left(\langle v\rangle\langle w\rangle+\left\langle v^{\prime} w^{\prime}\right\rangle+\left\langle\tau_{y z}\right\rangle-\nu \frac{d\langle v\rangle}{d z}\right) & =0, \\
\frac{d}{d z}\left(\langle w\rangle\langle w\rangle+\left\langle w^{\prime} w^{\prime}\right\rangle+\left\langle\tau_{z z}\right\rangle+\langle p\rangle\right) & =0 .
\end{aligned}
$$

$u^{\prime}=u-\langle u\rangle$ is the fluctuation part, and the external force $F$ is taken to be a constant pressure gradient in $x$ direction as the driving force. The no-slip boundary condition on the ground implies $\langle w\rangle=0$. We further assume a constant shear stress $\rho u_{*}^{2}$ on the ground, where $u_{*}$ is the friction velocity. Hence, the global force balance yields $u_{*}^{2}=F H$, where $H$ is the boundary layer height. The mean-flow momentum equations become

$$
\begin{aligned}
\langle w\rangle=\frac{d\langle w\rangle}{d z} & =0 \\
\left\langle u^{\prime} w^{\prime}\right\rangle+\left\langle\tau_{x z}\right\rangle-\nu \frac{d\langle u\rangle}{d z} & =(z-H) F, \\
\left\langle v^{\prime} w^{\prime}\right\rangle+\left\langle\tau_{y z}\right\rangle-\nu \frac{d\langle v\rangle}{d z} & =0 \\
\left\langle w^{\prime} w^{\prime}\right\rangle+\left\langle\tau_{z z}\right\rangle+\langle p\rangle & =p_{\text {wall }},
\end{aligned}
$$

where $p_{\text {wall }}$ is the mean pressure on the ground and is equal to the free-stream pressure. Equations (2.8b), (2.8c), and (2.8d) represent, in $x, y$, and $z$ directions, the force balance among the Reynolds shear stress, the SGS stress, the viscous shear stress, and the external forces.

\subsection{Energy budgets}

Let $k_{M F}=\frac{1}{2}\left\langle u_{i}\right\rangle\left\langle u_{i}\right\rangle$ denote the kinetic energy of the mean filtered velocity field, $k^{\prime}=$ $\frac{1}{2} u_{i}^{\prime} u_{i}^{\prime}$ the turbulent kinetic energy of the filtered velocity, $k_{f}=\frac{1}{2} u_{i} u_{i}$ the kinetic energy 
of the filtered velocity field, $k_{S G S}=\frac{1}{2} \tau_{i i}$ the SGS kinetic energy, and $k=\frac{1}{2} u_{i} u_{i}$ the filtered kinetic energy. The following relations hold

$$
\begin{aligned}
k & =k_{f}+k_{S G S}, \\
\left\langle k_{f}\right\rangle & =k_{M F}+\left\langle k^{\prime}\right\rangle .
\end{aligned}
$$

By multiplying Eqs. (2.5) by $u_{i}$ and adding all three component equations, we obtain the balance equation for $k_{f}$

$$
\begin{aligned}
\frac{\partial k_{f}}{\partial t}+\frac{\partial\left(k_{f} u_{j}\right)}{\partial x_{j}}= & -\frac{\partial}{\partial x_{j}}\left(p u_{j}+u_{i} \tau_{i j}-\nu \frac{\partial k_{f}}{\partial x_{j}}\right) \\
& -2 \nu S_{i j} S_{i j}+\tau_{i j} S_{i j}+F \cdot u,
\end{aligned}
$$

where $S_{i j}=\frac{1}{2}\left(\frac{\partial u_{i}}{\partial x_{j}}+\frac{\partial u_{j}}{\partial x_{i}}\right)$ is the filtered rate-of-strain tensor. A similar procedure where Eqs. (2.7) are mutiplied by $\left\langle u_{i}\right\rangle$ yields the balance equation for $k_{M F}$

$$
\begin{aligned}
0= & -\langle u\rangle \frac{d}{d z}\left(\left\langle u^{\prime} w^{\prime}\right\rangle+\left\langle\tau_{x z}\right\rangle\right)+\nu \frac{d^{2}}{d z^{2}}\left(\frac{\langle u\rangle^{2}}{2}\right) \\
& -\nu\left(\frac{d\langle u\rangle}{d z}\right)^{2}+F \cdot\langle u\rangle .
\end{aligned}
$$

Applying the averaging operator in Eq. (2.6) to Eq. (2.10), yields the balance equation for $\left\langle k_{f}\right\rangle$,

$$
\begin{aligned}
0= & \underbrace{-\frac{d}{d z}\left(\left\langle k_{f} w\right\rangle+\langle p w\rangle+\left\langle u_{i} \tau_{i z}\right\rangle-\nu \frac{d\left\langle k_{f}\right\rangle}{d z}\right)}_{\epsilon_{\nu}} \\
& \underbrace{-2 \nu\left\langle S_{i j} S_{i j}\right\rangle}_{\epsilon_{S G S}}+\underbrace{\left\langle\tau_{i j} S_{i j}\right\rangle}_{P_{F}}+\underbrace{F \cdot\langle u\rangle},
\end{aligned}
$$

and then subtract Eq. (2.11), giving the balance equation for $\left\langle k^{\prime}\right\rangle$,

$$
\begin{aligned}
0= & \underbrace{-\frac{d}{d z}\left(\left\langle k^{\prime} w^{\prime}\right\rangle+\left\langle p^{\prime} w^{\prime}\right\rangle+\left\langle u_{i}^{\prime} \tau_{i z}^{\prime}\right\rangle-\nu \frac{d}{d z}\left\langle k^{\prime}\right\rangle\right)}_{T^{\prime}} \\
& \underbrace{-\left\langle u^{\prime} w^{\prime}\right\rangle \frac{d\langle u\rangle}{d z}-\left\langle v^{\prime} w^{\prime}\right\rangle \frac{d\langle v\rangle}{d z}}_{P_{\text {shear }}^{\prime}} \underbrace{-2 \nu\left\langle S_{i j}^{\prime} S_{i j}^{\prime}\right\rangle}_{\epsilon_{\nu}^{\prime}}+\underbrace{\left\langle\tau_{i j}^{\prime} S_{i j}^{\prime}\right\rangle}_{\epsilon_{S G S}^{\prime}},
\end{aligned}
$$

where $T^{\prime}$ or $T$ accounts for the transport of velocity third-order moments, pressure, SGS stress, and viscous stress. $P_{\text {shear }}^{\prime}$ and $P_{F}$ denote the energy production of the mean shear and the external force, respectively. $\epsilon_{\nu}^{\prime}$ and $\epsilon_{S G S}^{\prime}$ correspond to the turbulent viscous and SGS dissipation. Note that the SGS dissipation $\epsilon_{S G S}$ controls the energy transfer between the filtered scale and the sub-grid scale, which plays a key role in the accuracy of the SGS model. Another source of dissipation is from the numerical discretization, referred to as numerical dissipation, which can be significant especially for coarse-grid simulations. In this report, the numerical dissipation is not addressed. 


\subsection{Subgrid-scale models}

To close Eqs. (2.5), it is necessary to model the SGS stress tensor $\tau_{i j}$, that is, $\tau_{i j}$ must be expressed as a function of the quantities $\mathbf{u}$ obtained in the simulations. The earliest and best known model linking $\tau$ and $\mathbf{u}$ is the Smagorinsky model [37]. The model has two parts. The first part is analogous to the eddy-viscosity model and describes the deviatoric component of $\tau_{i j}$, while the second part is similar to Prandlt's mixing-length model relating the eddy viscosity to the mean shear. The expression of the model is

$$
\begin{aligned}
\tau_{i j}^{d} & =-2 \nu_{S G S} S_{i j}, \\
\nu_{S G S} & =\left[C_{S} \Delta\right]^{2}|S|,
\end{aligned}
$$

where $\nu_{S G S}$ denotes the SGS viscosity, and $|S|=\left(2 S_{i j} S_{i j}\right)^{1 / 2}$ is the magnitude of the rate-of-strain tensor. $C_{S}$ is the Smagorinsky constant, and $C_{S} \Delta$ denotes a subgrid length scale. Usually, $\Delta$ is taken to be the power average of the grid sizes in all directions, $\Delta=\left(\Delta_{x} \Delta_{y} \Delta_{z}\right)^{1 / 3}$. The simplicity of this SGS model enables easy implementation. However, it is well known that this model is too dissipative in the near-wall region, because the subgrid length scale is not constant but depends on the distance from the wall.

A more elaborate model is called one-k-equation-eddy (oneEqEddy) model [10], which takes into account the history and non-local effects. In this model, SGS viscosity is defined as

$$
\nu_{S G S}=C_{k} k_{S G S}^{1 / 2} \Delta
$$

and SGS kinetic energy $k_{S G S}$ is solved by the transport equation

$$
\partial_{t} k_{S G S}+\mathbf{u} \nabla k_{S G S}=\left(\nu_{S G S}+\nu\right) \Delta k_{S G S}+2 \nu_{S G S}|S|^{2}-\frac{C_{\epsilon} k_{S G S}^{3 / 2}}{\Delta}
$$

The terms in the left-hand side of Eq. (2.16) denote the time change and convection by the resolved field $\mathbf{u}$, respectively. In the right-hand side, the three terms correspond to the viscous diffusion, the gradient diffusion (the energy transfer between the filtered and sub-grid scales) and the dissipation. The constants have typical values $C_{k}=0.094$ and $C_{\epsilon}=1.048$.

Many other SGS models exist, including dynamic models, similarity models, and gradient models. A comprehensive review on LES models can be found in [22]. Widely used in meteorology applications [23] and practically showing advantages over many other models [10], the oneEqEddy model is chosen for our simulations. 


\section{Simulation specifications}

To save computational costs and to be comparable with the wind tunnel experiments, the geometric scale for the simulation is taken to be 1:100. Assuming that the height of the atmospheric boundary layer is of the order of $1 \mathrm{~km}$, the domain height $H$ we are simulating is taken to be $10 \mathrm{~m}$. It is further assumed that the terrain roughness corresponds to open terrain exposure, that is, the prototype roughness length is assumed to be $0.03 \mathrm{~m}$ [35], so that the model counterpart is $z_{0} \simeq 0.0003 \mathrm{~m}$. The kinematic viscosity of the air is taken to be $\nu=1.455 \times 10^{-5} \mathrm{~m}^{2} / \mathrm{s}$ (at temperature $15^{\circ} \mathrm{C}$ and at sea level). The velocity scale is taken to be unity, and the target mean velocity at the top, $u_{\text {ref }}$, is $30 \mathrm{~m} / \mathrm{s}$. The surface friction velocity can then be estimated from the logarithmic velocity profile, $u_{*}=\frac{\kappa u_{\mathrm{ref}}}{\ln \left(H / z_{0}\right)}=1.181 \mathrm{~m} / \mathrm{s}$. The Von Karman constant $\kappa$ is assumed to be 0.41 . Table 3.1 is a summary of the parameters in the simulations. Note that the dynamics in model-scale flow $(H=10 \mathrm{~m})$ and in full-scale flow $(H=1000$ $\mathrm{m})$ are similar if the molecular viscosity effects are negligible, since the non-dimensional governing equations (2.5) for both cases are identical.

\begin{tabular}{lc}
\hline \hline Parameter & Value \\
\hline Height $[\mathrm{m}]$ & $H=10$ \\
Roughness length $[\mathrm{m}]$ & $z_{0}=0.0003$ \\
Reference velocity $[\mathrm{m} / \mathrm{s}]$ & $u_{\text {ref }}=30$ \\
Reference height $[\mathrm{m}]$ & $z_{\text {ref }}=10$ \\
Air viscosity $\left[\mathrm{m}^{2} / \mathrm{s}\right]$ & $\nu=1.455 \times 10^{-5}$ \\
von Karman constant & $\kappa=0.41$ \\
expected friction velocity $[\mathrm{m} / \mathrm{s}]$ & $u_{*}=1.181$ \\
\hline \hline
\end{tabular}

Table 3.1: Parameters in the simulations.

\subsection{Geometry, boundary and initial conditions}

The geometry and the coordinate system of the computational domain are shown in Fig. 3.1. The size in the horizontal directions is $20 \mathrm{~m} \times 20 \mathrm{~m}$. A periodic boundary condition is imposed in the horizontal directions. For velocity, no-slip boundary condition is applied on the ground, while the slip condition is applied at the top. For pressure field a zero-gradient boundary condition is imposed at both the ground and the top. The instantaneous shear stress wall function, originally introduced by Schumann [34] based on local equilibrium in the near-wall region, is applied at the ground level, as follows.

$$
\begin{aligned}
& \tau_{x z, \text { wall }}=-u_{*}^{2} \frac{u_{\Delta}}{\left|\mathbf{u}_{\Delta}\right|}=-\left[\frac{\kappa\left|\mathbf{u}_{\Delta}\right|}{\ln \left(z_{\Delta} / z_{0}\right)}\right]^{2} \frac{u_{\Delta}}{\left|\mathbf{u}_{\Delta}\right|}, \\
& \tau_{y z, \text { wall }}=-u_{*}^{2} \frac{v_{\Delta}}{\left|\mathbf{u}_{\Delta}\right|}=-\left[\frac{\kappa\left|\mathbf{u}_{\Delta}\right|}{\ln \left(z_{\Delta} / z_{0}\right)}\right]^{2} \frac{v_{\Delta}}{\left|\mathbf{u}_{\Delta}\right|} .
\end{aligned}
$$

Distinguished from the filter width $\Delta$, the subscript $\Delta$ indicates the center position of the first grid level above the ground where the velocity is considered. $\left|\mathbf{u}_{\Delta}\right|=$ 
$\sqrt{\left\langle u_{\Delta}\right\rangle^{2}+\left\langle v_{\Delta}\right\rangle^{2}}$ is the magnitude of the horizontally averaged velocity at $z=z_{\Delta}$. Note that $u_{*}$ is not a constant value but instead obtained from the horizontally averaged velocity at each time step by assuming a logarithmic mean profile near the ground [13]. In Schumann's original paper [34], the momentum balance is expressed as $u_{*}^{2}=F H$, which does not account for history effects or fluctuations. The boundary conditions used in the simulations will subsequently be shown to yield a mean profile closer to the target profile. Actually, the choice of wall function depends on numerous factors like mesh, grid resolution, and numerical scheme. Lee et al. [18] showed that mean shear stress wall function predicts accurately the mean profile and low-order statistics even with coarse-grid resolutions. In addition, the location where the shear stress condition is imposed can influence significantly the numerical errors [15]. Here we choose a wall funtion that is commonly used in the meteorology community (for other types of wall functions, refer to [28]).

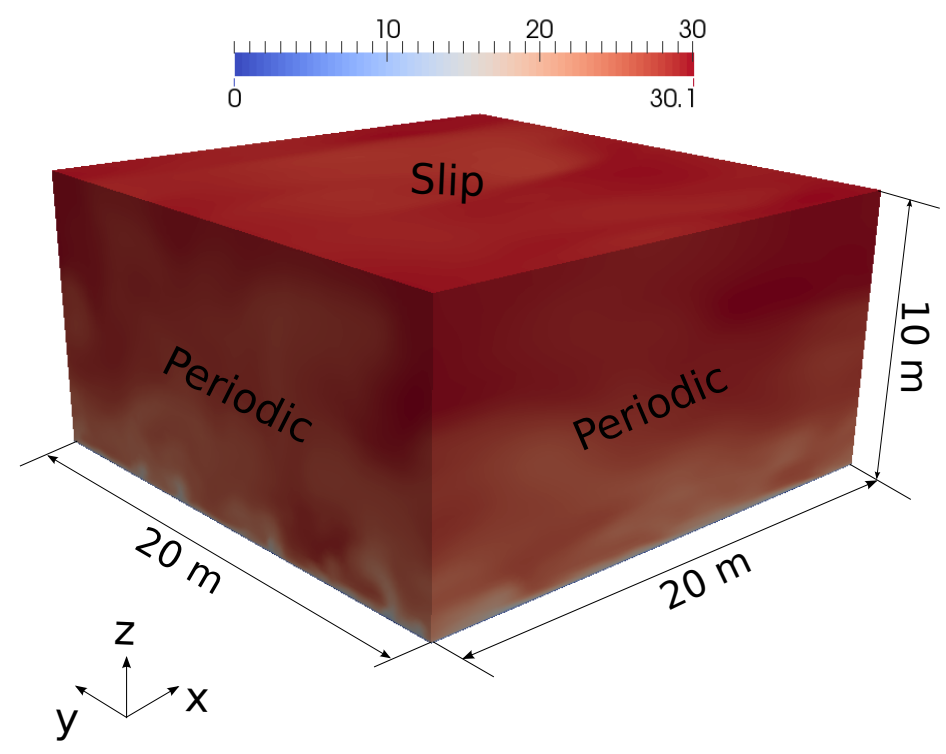

Figure 3.1: Coordinates and geometry of the computations. The color map corresponds to instantaneous velocity magnitude.

Following the proposal in [33], the initial condition for velocity consists of a laminar logarithmic profile and a small perturbation near the ground. The perturbation is applied only to the horizontal velocity components $\left(u_{0}, v_{0}\right)$, as follows:

$$
\begin{aligned}
& u_{0}(y, z)=\frac{u_{*}}{\kappa} \ln \left(\frac{z}{z_{0}}\right)+A_{u}(z) \sin (\beta y)(1+\lambda), \\
& v_{0}(x, z)=A_{v}(z) \cos (\alpha x)(1+\lambda) .
\end{aligned}
$$

$A_{u}(z)$ and $A_{v}(z)$ are amplitude function and take the form of $z e^{-\sigma z^{2}}$. This confines the perturbation to the near-wall region if a proper attenuation factor $\sigma$ is specified. $\alpha$ and $\beta$ are the wavenumbers of the perturbation while the factor $\lambda$ is a small $( \pm 20 \%$ of the perturbation amplitude) random component to break the symmetry. The amplitude of the perturbation part is lower than $5 \%$ of the mean flow. This form of perturbation 
creates streaky flow structures (low- and high-speed flow patches) which favor the development of turbulence, and hence significantly shorten the initial relaxation time. The initial pressure is set zero everywhere.

\subsection{OpenFOAM: a finite-volume numerical solver}

All the simulations are performed with the open source computational fluid dynamics toolbox OpenFOAM (version 2.3.0) [27]. Compared to other commercial CFD solvers, OpenFOAM has the advantage of full flexibility with control of all stages from code development to postprocessing. We employ a large time-step transient solver in OpenFOAM toolbox for incompressible flow using PIMPLE (merged PISO-SIMPLE) algorithm. PIMPLE, SIMPLE, and PISO are different algorithms in the general operatorsplitting approach [14]. The solver used in this report is a modified form of the standard solver pimpleFoam. An external constant force $F$ is added to the $x$-component differential equation, and most of the horizontally averaged statistics are generated in runtime. This solver makes it possible to dynamically adjust the time step in runtime based on the specified maximum Courant-Friedrichs-Lewy (CFL) number (CFL $=0.5$ in the simulations), which reduces the initial relaxation time. The time discretization is performed by the implicit Crank-Nicolson scheme with a coefficient of 0.5. The Gaussian-type schemes are chosen for the spatial (gradient, divergence, and laplacian) discretization. The Poisson equation for pressure is solved by GAMG (Generalized Geometric-Algebraic Multi-Grid) algorithm, while the linear equation for velocity is solved by PBiCG algorithm (preconditioned bi-conjugate gradient solver for asymmetric matrices). Linear interpolation is used to obtain the physical quantities at the surface centers of the cells.

Structured mesh is utilized in the simulations due to the simple geometry of the computational domain. The domain size is $L_{x} \times L_{y} \times L_{z}=20 \mathrm{~m} \times 20 \mathrm{~m} \times 10 \mathrm{~m}$, with a resolution of $150 \times 150 \times 75$ cells. The grid is uniform, equidistant in all directions, which removes the commuting errors of the LES filtering operator for non-uniform grids. The grid size in $z$-direction is $\Delta z=0.0133 H$, which is relatively coarse compared to the grid size used in direct numerical simulations. 


\section{Horizontally-averaged flow statistics}

We perform simulations until the statistical states are stationary, and then we sample data for $300 \mathrm{~s}$, equivalent to approximately 450 turnovers at $z=0.1 \mathrm{H}$. Figure 4.1 shows the contours of the magnitude of the instantaneous filtered velocity fluctuations at different heights. The streaky turbulent structures dominate near the ground, whereas at the top the coherent structures are much larger. The magnitudes of the fluctuations indicate that the turbulence intensity peaks in the near-ground region and decreases towards the top. To adequately evaluate the quality of the numerical simulations, mean velocity and turbulence statistics are further analyzed in this section.
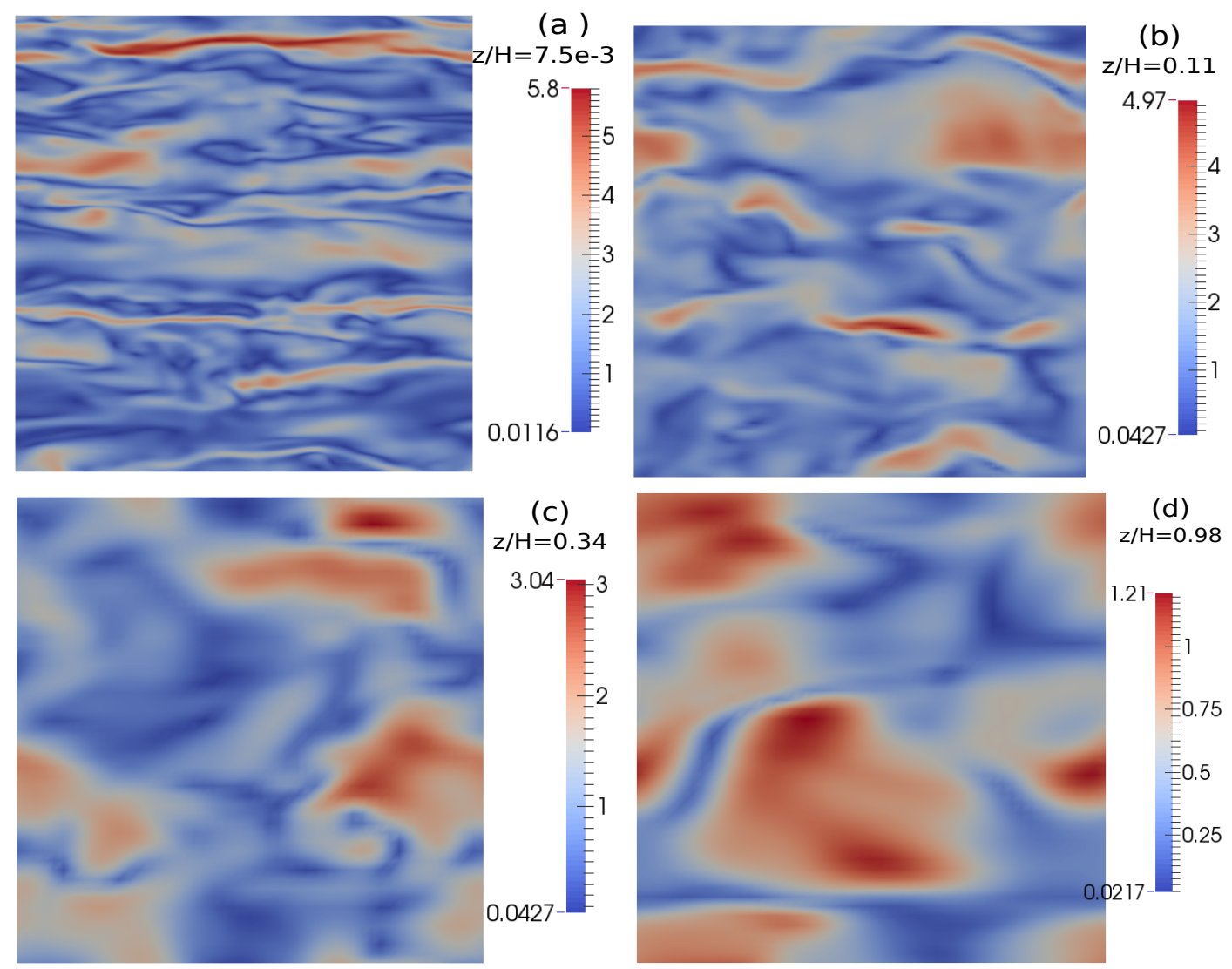

Figure 4.1: Contours of the magnitude of the filtered velocity fluctuactions at different heights: (a) $z / H=0.0075$, (b) $z / H=0.11$, (c) $z / H=0.34$, (d) $z / H=0.98$. The wind direction is from left to right.

\subsection{Mean velocity}

In atmospheric boundary layer (ABL) flow, the mean wind velocity follows approximately a logarithmic profile, $\frac{\langle u\rangle}{u_{*}}=\frac{1}{\kappa} \ln \left(\frac{z}{z_{0}}\right)$. The underlying assumptions are the Boussinesq approximation and the Prandtl's mixing length hypothesis. The Boussinesq approximation connects the Reynold stress to the mean shear via the eddy viscosity $\nu_{T}$, 
$-\left\langle u^{\prime} w^{\prime}\right\rangle=\nu_{T} \frac{d\langle u\rangle}{d z}$, while $\nu_{T}$ is further estimated by surface velocity $u_{*}$ and the Prandtl's mixing length scale $\kappa z, \nu_{T}=u_{*} \kappa z$. In the bulk flow, the Reynolds stress is balanced approximately by the wall shear, which leads to the equation $-\rho u_{*}^{2}=-\rho u_{*} \kappa z \frac{d\langle u\rangle}{d z}$. The above log-law profile is obtained by solving this equation and considering the boundary condition at the aerodynamic roughness height $z_{0}$. A different way to obtain the logarithmic profile is based on the classical asymptotic approach [4, 39], which is summarized in [36]. Note that this logarithmic profile holds for the whole ABL height due to the constant external pressure gradient [36].

Figure 4.2 (left) shows the mean wind velocity, $\langle u\rangle$, as a function of the dimensionless height, $z / H$. The target logarithmic profile is also plotted in dashed line. The profile from the simulation deviates by about $10 \%$ from the target profile. The deviation should be largely attributed to the inaccuracy of the SGS model in the near-ground region, which will be later discussed in this paper. Near the top, the flattened profile introduces a strong $z$ component of the velocity gradient, which is in contradiction to the slip boundary condition. The slip boundary condition imposes zero normal gradient in the wall-parallel velocity components. The underlying reason of this inconsistency is still unclear. The mean velocity $\langle v\rangle$ and $\langle w\rangle$ are negligibly small.

As is shown by the mean-flow momentum equations (2.8) and in the energy budget equation (2.13), the velocity gradient is much more relevant for the flow dynamics than the velocity itself. We introduce the nondimensional mean velocity gradient $\Phi=\frac{\kappa z}{u_{*}} \frac{d\langle u\rangle}{d z}$, which is equal to unity for the target logarithmic profile. The derivative is estimated by the central finite difference method. The stencil consists of 9 points in the middle and gradually decreasing number of points towards both ends. The vertical profile of $\Phi$ is shown in Fig. 4.2 (right). Near the ground $(z / H<0.1)$ and at the top $(z / H>0.9)$, the simulation value departs from the target profile. The log-layer mismatch (also referred to as "overshooting") problem near the ground is well known in LES simulations. However, since top part of the boundary layer is not as important as the near-wall region where turbulence arises, the mismatch at the top is rarely mentioned in previous publications.
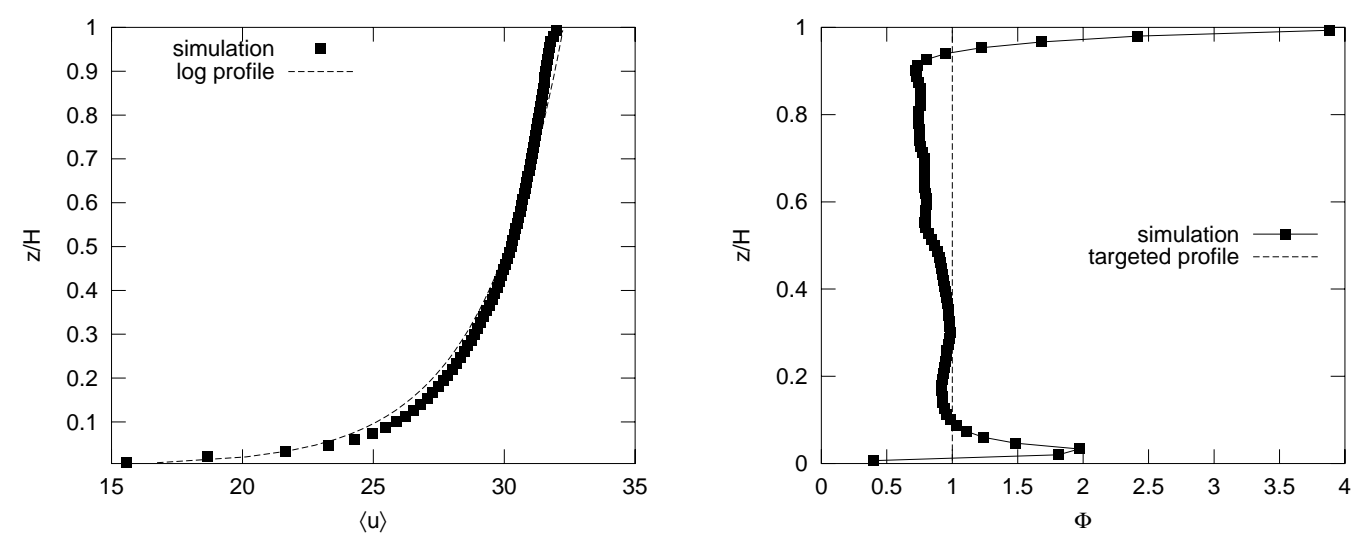

Figure 4.2: Vertical profiles of the mean streamwise velocity $\langle u\rangle$ (left) and the nondimensional mean velocity gradient $\Phi=\frac{\kappa z}{u_{*}} \frac{d\langle u\rangle}{d z}$ (right). The dashed lines are the logarithmic profiles. 


\subsection{Velocity variances}

Velocity variance is a measure of the strength of turbulent fluctuations. Let $\sigma_{u}^{2}$ denote the averaged variance of the streamwise filtered velocity, namely, $\sigma_{u}^{2}=\left\langle u^{\prime 2}\right\rangle$. $\sigma_{v}^{2}$ and $\sigma_{w}^{2}$ are the variances of the fluctuations in $y$ and $z$ directions. Our results are plotted in Fig. 4.3 (left). The variances are normalized by $u_{*}^{2}$. It is shown that the variance in the streamwise direction is much higher than in other two directions. The shape and the magnitude of the profiles are consistent with the analytical expressions from the similarity theory [38] and the previously published results (see $[31,3,5])$.

The averaged turbulent kinetic energy $\left\langle k^{\prime}\right\rangle$ is $\left\langle k^{\prime}\right\rangle=\left(\sigma_{u}^{2}+\sigma_{v}^{2}+\sigma_{w}^{2}\right) / 2$. As shown in Fig. 4.3, the vertical profile of the normalized $\left\langle k^{\prime}\right\rangle$ follows a logarithmic profile, $\left\langle k^{\prime}\right\rangle \sim$ $\ln (z / H)$, except for the near-ground region. Since turbulence arises from the interaction between the flow and the ground, the turbulent energy is largest near the ground and is transported upwards. Note that the decreasing in magnitude near the ground here is not due to the molecular viscosity effect but mainly to the strong SGS dissipation which extracts energy from the filtered motions to the residual motions. The grid size used in this report is too coarse to activate the molecular viscosity effect.
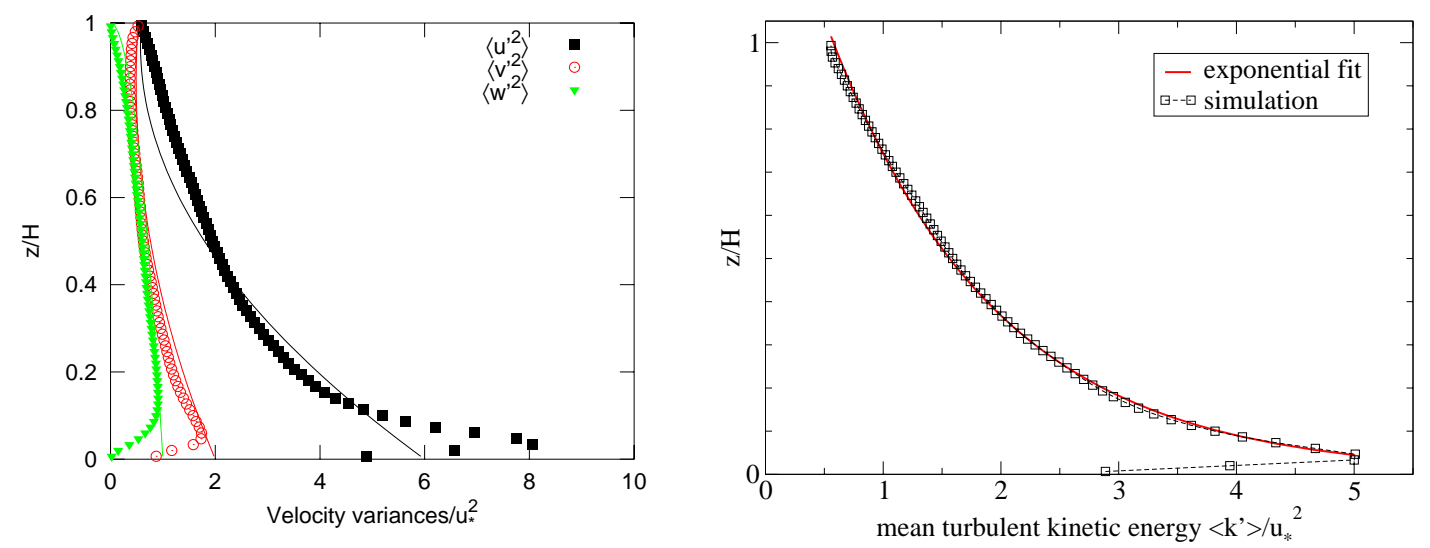

Figure 4.3: Vertical profiles of the normalized variance of the filtered velocity (left) and the normalized turbulent kinetic energy (right). The solid lines in the left panel are analytical expressions from similarity theory [38]. The red bold line in the right panel is the exponential fitting curve, $z / H \simeq 1.5 \mathrm{e}^{-0.7\left\langle k^{\prime}\right\rangle}$, and hence $\left\langle k^{\prime}\right\rangle \sim \ln (z / H)$.

\subsection{Reynolds stresses}

Reynolds stresses physically represent the momentum transportation in the flow. As shown by Eq. (2.8), the total stresses in the streamwise direction are balanced by the external pressure gradient, varying linearly with height as $u_{*}^{2}(z / H-1)$. Since the molecular viscous effect is negligible (2-3 order of magnitude smaller), the sum of Reynolds and SGS stress is thus a linear function of the height $z$. Figure 4.4 (left) shows the normalized mean Reynolds and SGS stress in the streamwise direction. Their sum is in agreement within $95 \%$ with the theoretical profile (dotted line) at $z / H>0.1$. The 
SGS stress is dominant at the near-wall region and is apparently too large (the lowest point not shown in the figure). On the other hand, the Reynolds stresses in other two directions are very small compared to $\left\langle u^{\prime} w^{\prime}\right\rangle$ (see the right panel in Fig. 4.4).
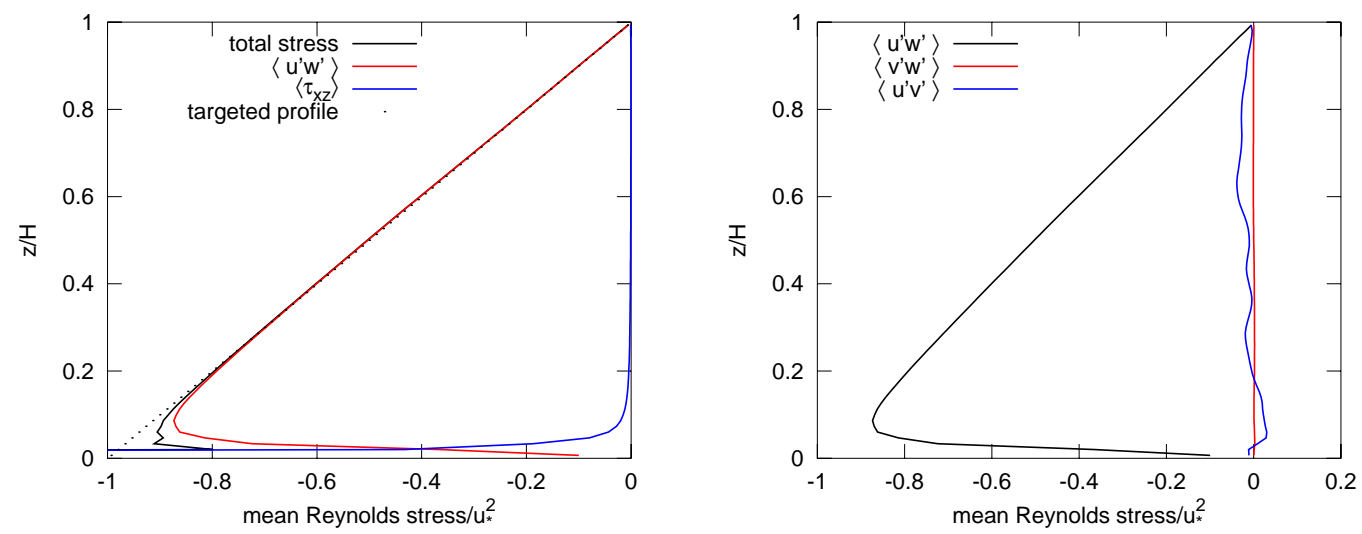

Figure 4.4: Vertical profiles of the normalized Reynolds stresses. (left) $\left\langle u^{\prime} w^{\prime}\right\rangle$ and the SGS stress $\left\langle\tau_{x z}\right\rangle$ and (right) $\left\langle u^{\prime} w^{\prime}\right\rangle,\left\langle v^{\prime} w^{\prime}\right\rangle$, and $\left\langle u^{\prime} v^{\prime}\right\rangle$. The dotted line in the left panel is the theoretical profile of the total streamwise stress.

\subsection{Fluxes of variances and Reynolds stresses}

The turbulent fluxes of velocity variances and Reynolds stresses are third-order moments of turbulence. The normalized vertical fluxes of velocity variances and Reynolds shear stresses are shown in Fig. 4.5. The vertical fluxes of variances are all positive, indicating upward transportation of turbulent kinetic energy. Although the fluxes of $\left\langle u^{\prime} w^{\prime}\right\rangle$ is negative, it represents an upward transportation, since the Reynold stress itself is negative. The fluxes of other two Reynolds stress components are negligibly small. These observations are qualitatively consistent with the results in published results [3]. They also imply that turbulence firstly arises near the ground, then is transported upwards and finally affects the upper bulk flow.

\subsection{SGS kinetic energy, SGS viscosity, and SGS stress}

To examine the accuracy of the SGS model, we now analyze the statistically averaged SGS kinetic energy $k_{S G S}$, viscosity $\nu_{S G S}$, and stress $\tau_{x z}$. According to the definition in oneEqEddy model, the SGS viscosity and stress depend largely on the SGS kinetic energy $k_{S G S}$ which is obtained numerically from the transport equation (2.16). In the statistically steady state, the time derivative, advection, and molecular diffusion are negligibly small. The simplified equation can be interpreted as a balance between the gradient diffusion and the dissipation, $2 \nu_{S G S}|S|^{2} \simeq C_{\epsilon} k^{3 / 2} / \Delta$, which actually leads to the original Smagorinsky model $\nu_{S G S}=\left(C_{s} \Delta\right)^{2}|S|$ with $C_{s}=\left(C_{k}^{3} / C_{\epsilon}\right)^{1 / 4}$. By virtue of the definition of $\nu_{S G S}$ (Eq. (2.15)) and the logarithmic mean velocity profile, the SGS kinetic energy is $k_{S G S} \sim\left|\frac{d u}{d z}\right|^{2} \sim z^{-2}$. In the simulations, the mean SGS kinetic energy 

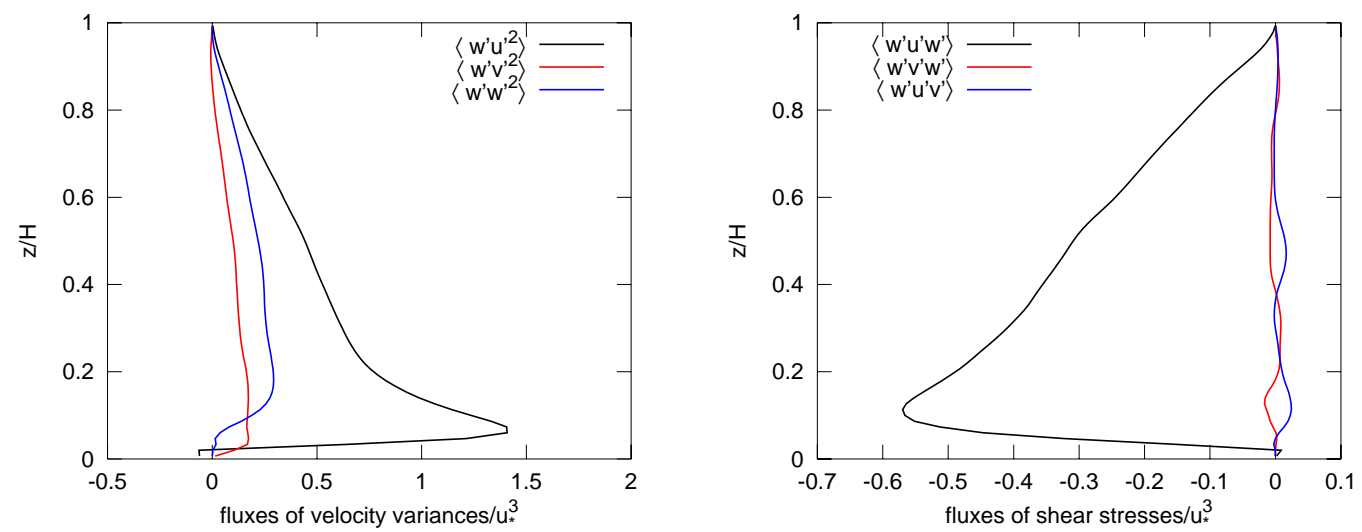

Figure 4.5: Vertical profiles of the normalized turbulent fluxes of velocity variances $\left\langle w^{\prime} u^{\prime 2}\right\rangle,\left\langle w^{\prime} v^{\prime 2}\right\rangle,\left\langle w^{\prime 3}\right\rangle$ (left) and Reynolds stresses $\left\langle w^{\prime} u^{\prime} w^{\prime}\right\rangle,\left\langle w^{\prime} v^{\prime} w^{\prime}\right\rangle,\left\langle w^{\prime} u^{\prime} v^{\prime}\right\rangle$ (right).

follows a power law profile, as shown by the black line in Fig. 4.6. However, the power exponents in all simulations range from -1.8 to -1.4 . This deviation may be attributed to the transportation terms. On the other hand the SGS energy near the ground is curved towards larger values, which leads to larger $\nu_{S G S}$ and SGS dissipation near the ground.

The SGS viscosity $\left\langle\nu_{S G S}\right\rangle$ and stress $\left\langle\tau_{x z}\right\rangle$ are also plotted in Fig. 4.6. As expected, they are power-law funtions of the height, which is also consistent with the calculations based on Eqs. (2.15) and (2.14). Note that in Fig. $4.6\left\langle k_{S G S}\right\rangle$ and $\left\langle\tau_{x z}\right\rangle$ are normalized by $u_{*}^{2}$ while $\left\langle\nu_{S G S}\right\rangle$ is normalized by $u_{*} H$.

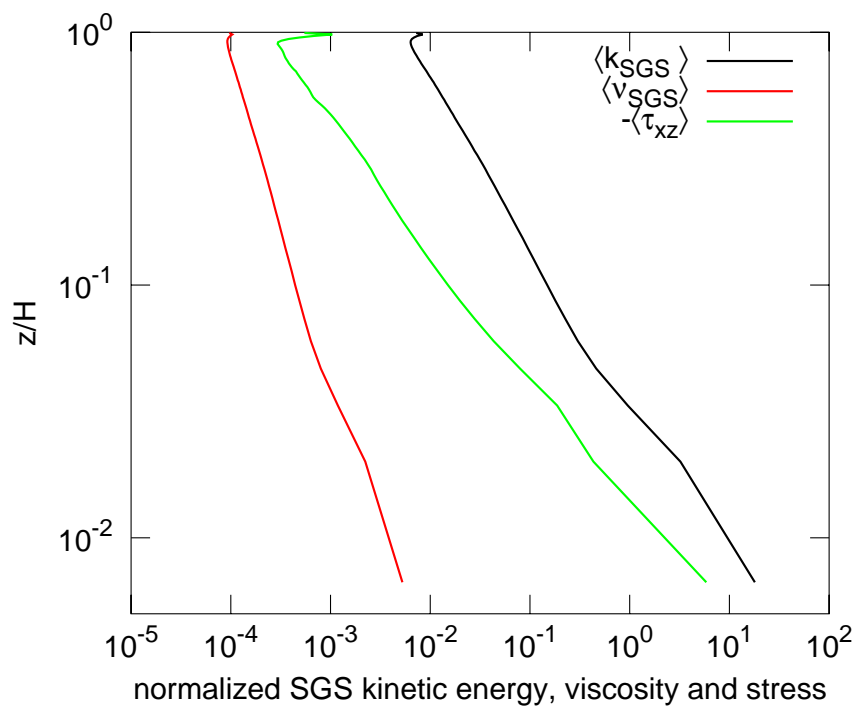

Figure 4.6: Vertical profiles of the SGS kinetic energy (black), SGS viscosity (red), and SGS stresses (green). $\left\langle k_{S G S}\right\rangle,\left\langle\nu_{S G S}\right\rangle$, and $\left\langle\tau_{x z}\right\rangle$ are normalized by $u_{*}^{2}, u_{*} H$, and $u_{*}^{2}$, respectively. The axes are in logarithmic scale. 


\subsection{Turbulent kinetic energy budget}

According to the budget equation (2.13) for the mean turbulent kinetic energy, the sum of transportation terms $T^{\prime}$, shear production $P_{\text {shear }}^{\prime}$, turbulent viscous dissipation $\epsilon_{\nu}^{\prime}$, , and SGS dissipation $\epsilon_{S G S}^{\prime}$ vanishes. Neglecting the viscous terms, the contributions of other terms as a function of height are shown in Fig. 4.7 (left). At $z / H>0.1$, the transport terms are very small, and the shear production is balanced by the turbulent SGS dissipation, whereas at $z / H<0.1$ the transport terms, dominated by the SGS transport, become overwhelmingly large due to the inaccurate LES model.
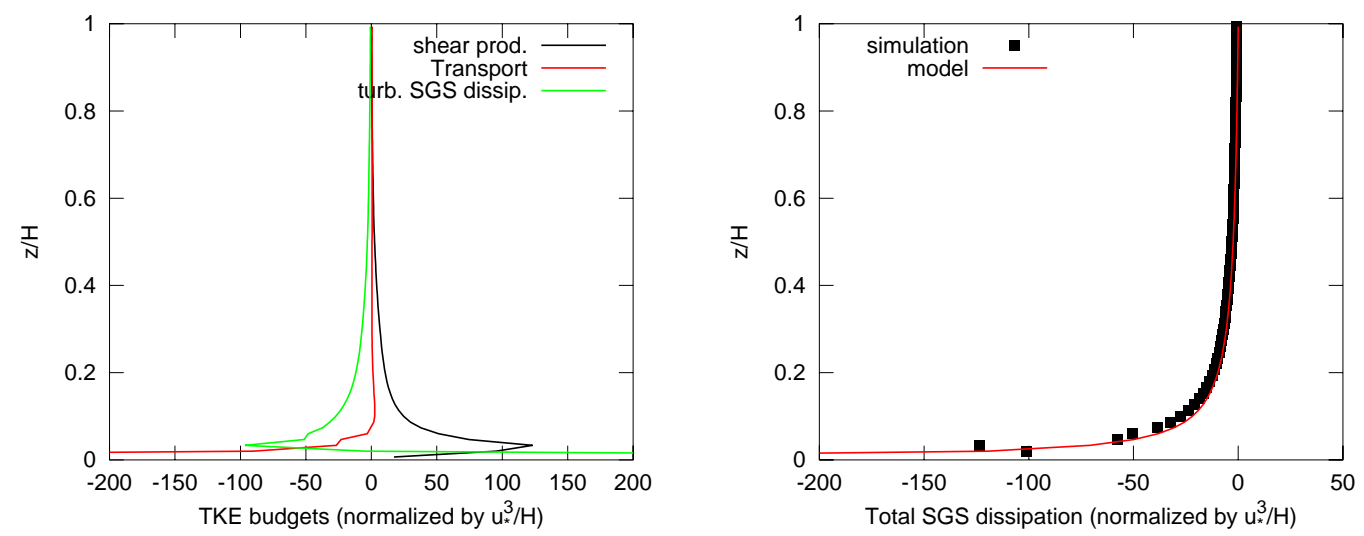

Figure 4.7: Vertical profiles of the contributions to the mean TKE from shear production, turbulent SGS dissipation, transport terms (left), and total SGS dissipation and its model (right). The $\mathrm{X}$-axis is normalized by $u_{*}^{3} / H$.

Now we consider the total SGS dissipation which controls the energy transfer between the filtered and SGS flows. The total SGS dissipation consists of two parts: one from the turbulent SGS dissipation $\epsilon_{S G S}^{\prime}$ and the other from the mean-flow SGS dissipation $\epsilon_{S G S}^{M F}=\left\langle\tau_{i j}\right\rangle\left\langle S_{i j}\right\rangle$. By making further use of the energy balance mentioned above and the mean-flow momentum equation (2.8), we have

$$
\begin{aligned}
\epsilon_{S G S} & =\epsilon_{S G S}^{\prime}+\epsilon_{S G S}^{M F} \\
& \simeq\left\langle u^{\prime} w^{\prime}\right\rangle \frac{d\langle u\rangle}{d z}+\left\langle\tau_{x z}\right\rangle \frac{d\langle u\rangle}{d z} \\
& \simeq u_{*}^{2}\left(\frac{z}{H}-1\right) \frac{d\langle u\rangle}{d z} \\
& \simeq \frac{u_{*}^{3}}{\kappa H}\left(1-\frac{1}{z / H}\right) .
\end{aligned}
$$

Inspired by the model proposed by Moeng and Sullivan [24], this model removes the factor $\Phi$, i.e., the dependence on the flow itself. The prediction from this model agrees with the results obtained from the simulations, as shown in Fig. 4.7 (right). 


\section{Probability density function, autocovariance, spec- tra, and spatial coherence}

Although not frequently dealt with by theoretical fluid dynamicists and meteorologists, these statistics are significant in engineering applications, e.g., for the prediction of peak wind loading on ground-based or aircraft structures.

The time series of the velocity components at one point are shown in Fig. 5.1 (top left). The coordinates of this point are $(H, H, 0.1 H)$. The time series are sampled for $300 \mathrm{~s}$ starting from $t=600 \mathrm{~s}$, where the flow is statistically stationary. The $u$ component displays strong fluctuations with large troughs and relatively low peaks. Its asymmetry is clearly reflected in the probability density function(PDF) of the fluctuation $u^{\prime}$, whereas the fluctuation $v^{\prime}$ and $w^{\prime}$ follows approximately the normal (Gaussian) distributions (Fig. 5.1). Similar asymmetry is observed at different heights and is probably attributed to non-Gaussian turbulent "patches" observed in the lower part of the boundary layer [1].
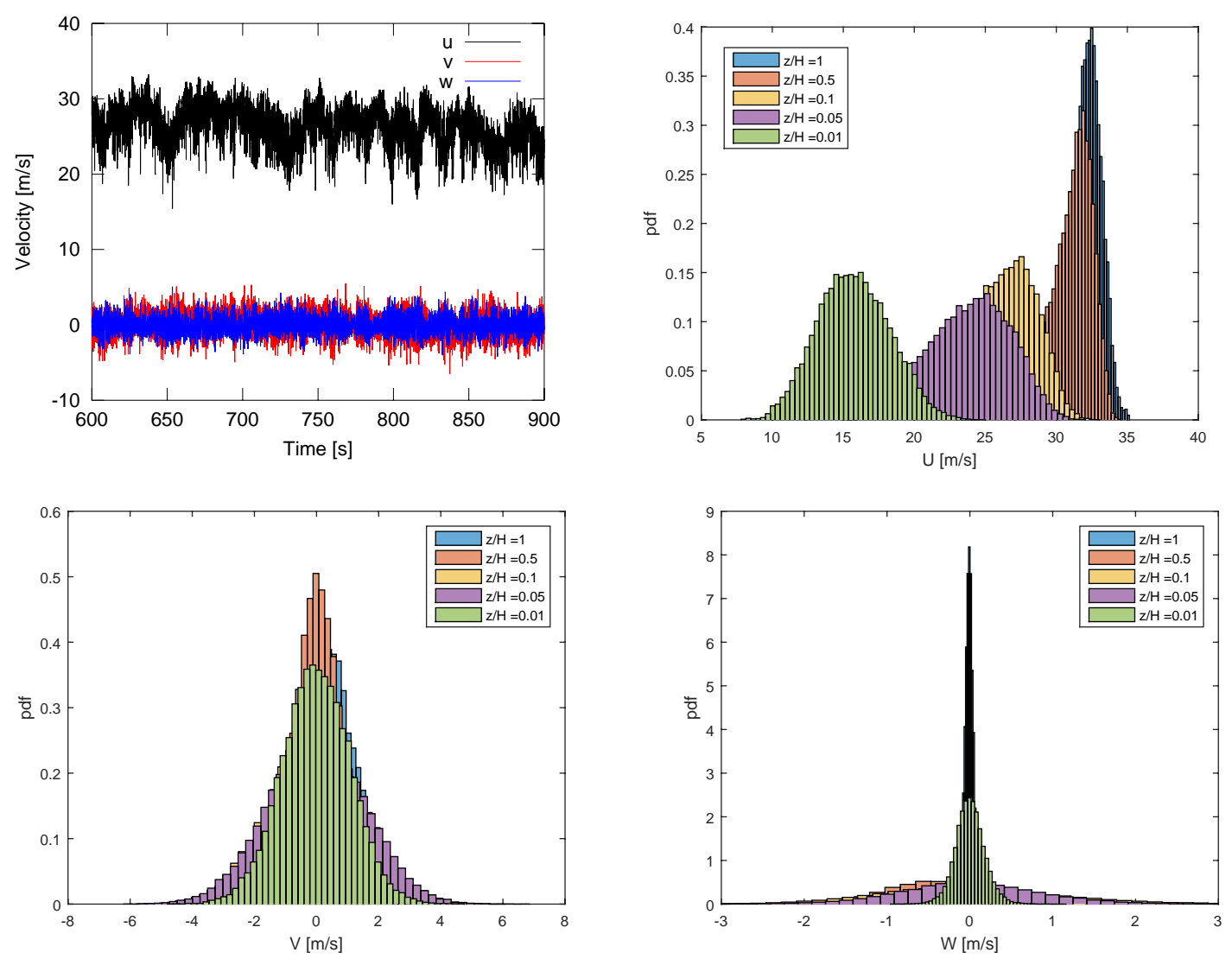

Figure 5.1: (Top left) Time series of velocity at a single point at $z / H=0.1$; (Others) PDF of velocity components at different heights.

Other important statistical quantities are the auto-covariance and power spectral density. The auto-covariance provides information on the extent in time to which the velocity fluctuation is correlated to itself. As a function of time lag mathematically, 
it is a mean product of two fluctuating components measured at the same point but at different times, $R_{u u}(\tau)=\frac{1}{T} \int_{0}^{T} u^{\prime}(t) u^{\prime}(t+\tau) d t$. For $\tau=0, R_{u u}(0)=\sigma_{u}^{2}$ becomes the variance. The auto-correlation is the normalized auto-covariance, $C_{u u}=R_{u u} / \sigma_{u}^{2}$. Figure 5.2 (left) shows the auto-correlation of the velocity components $(u, v, w)$, which are fitted by exponential functions with exponents $-5.5,-17.17$, and -18.03 , respectively. As the time lag increases, the auto-correlation tends to zero.
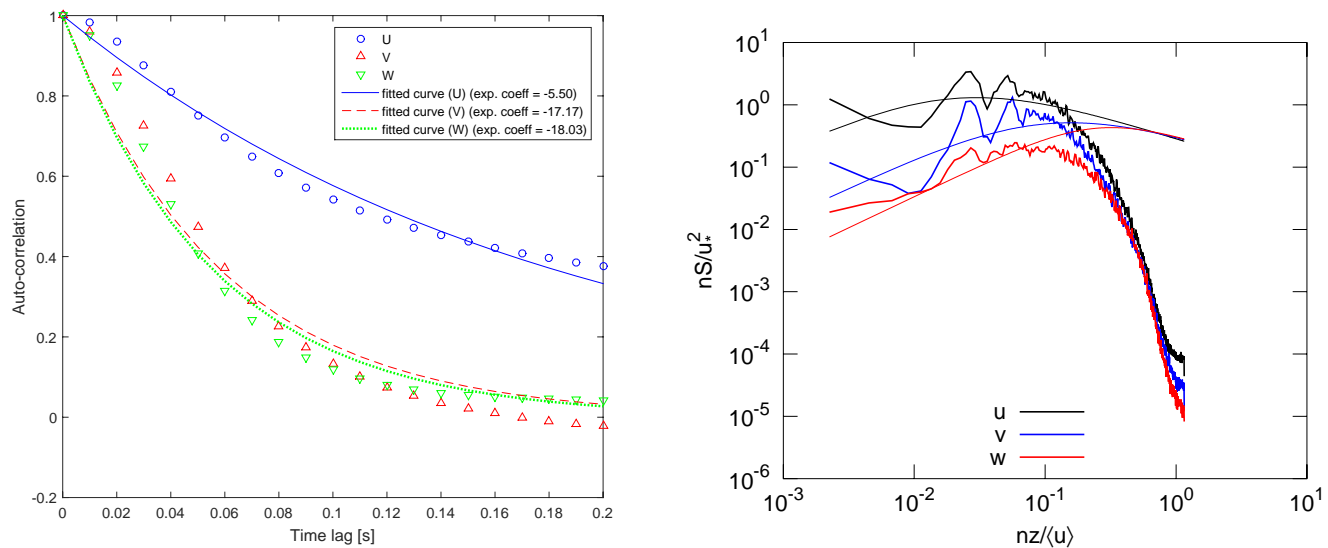

Figure 5.2: (left) Autocorrelation function of velocity components and the corresponding exponential fits; (right) spectral density function of velocity fluctuations. The smooth thin lines are empirical formula for spectra of different velocity component.

On the right panel of Fig. 5.2, the nondimensional power spectra $f=\frac{n S_{u}}{u_{*}^{2}}\left(u_{*}\right.$ is the target friction velocity) is plotted as a function of nondimensional frequency $\frac{n z}{\langle u\rangle}$. The power spectra is the Fourier transform of auto-covariance, that is, $S_{u}(n)=$ $\int_{-\infty}^{+\infty} 2 R_{u u} \cos (2 \pi n \tau) d \tau$. According to the Parseval's theorem $\sigma_{u}^{2}=\frac{1}{T} \int_{0}^{T} u^{2}(t) d t=$ $\int_{0}^{\infty} S_{u}(n) d n$, the power spectral represents the contribution of each frequency component to the velocity variance at a single point, and plays a key role in turbulence theory and engineering applications. For neutrally stratified flows, the power spectra of each flow component follow approximately the modified Kaimal spectra model in the part of atmospheric boundary layer [35] in which the log law holds, that is,

$$
\begin{aligned}
\frac{n S_{u}}{u_{*}^{2}} & =\frac{200 f}{(1+50 f)^{5 / 3}} \\
\frac{n S_{v}}{u_{*}^{2}} & =\frac{15 f}{(1+9.5 f)^{5 / 3}} \\
\frac{n S_{w}}{u_{*}^{2}} & =\frac{3.36 f}{1+10 f^{5 / 3}}
\end{aligned}
$$

It is shown in Fig. 5.2 (right) that the power spectra are consistent with the Kaimal expressions in the low frequency range and drop rapidly as the cutoff frequency is approached. Note the approximate similarity between the shapes of the spectra in Fig. 5.2 and in Fig. 2 of Coleman's paper [9]. 
To evaluate the correlation between two points in space and in time, cross-covariance and cross-spectra are appropriate measures. The cross-spectrum $S_{u_{1} u_{2}}$ is the Fourier transform of the cross-covariance $R_{u_{1} u_{2}}(\Delta r, \tau)$ and is a complex quantity consisting of a symmetric part $S_{u_{1} u_{2}}^{C}$ and an anti-symmetric part $S_{u_{1} u_{2}}^{Q}$, namely, $S_{u_{1} u_{2}}=S_{u_{1} u_{2}}^{C}+i S_{u_{1} u_{2}}^{Q}$. The coherence of two signals is defined

$$
\gamma_{u_{1} u_{2}}^{2}(\Delta r, n)=\frac{\left(S_{u_{1} u_{2}}^{C}\right)^{2}+\left(S_{u_{1} u_{2}}^{Q}\right)^{2}}{S_{u_{1} u_{1}} S_{u_{2} u_{2}}} .
$$

The coherence is a function of the distance between the two points $\Delta r$ and the frequency $n$. If two points are along the wind direction (x-axis), the square root of the coherence can be approximated by the exponential function of frequency, $\gamma_{u_{1} u_{2}} \simeq e^{-c_{\gamma x} n \Delta x /\langle u\rangle}$ $[2,35] . c_{\gamma x}$ is a coefficient in the exponent in $x$ direciton.

In Fig. 5.3 (left), the coherence functions of two points at the same height but with different distances are plotted. They follow exponential functions of the frequency $n$. We further normalized the frequency by $\langle u\rangle / \Delta x$, and we found that all curves collapse on a single exponential curve, with a fitting parameter $c_{\gamma x}=0.46 \pm 0.01$. This value is about the same in all simulations.

Similar procedures are applied in the $y$ and $z$ direction. As shown in Fig. 5.4 and Fig. 5.5, the coherence functions are both exponential, and the exponents are $c_{\gamma y}=$ $13.48 \pm 0.43$ and $c_{\gamma z}=8.89 \pm 0.22$, respectively. These results indicate that the coherent structures have much larger size in the along-wind direction than in the cross-wind and vertical directions, which is consistent with the near-wall elongated streaky structures observed both in experiments and simulations [32, 33].
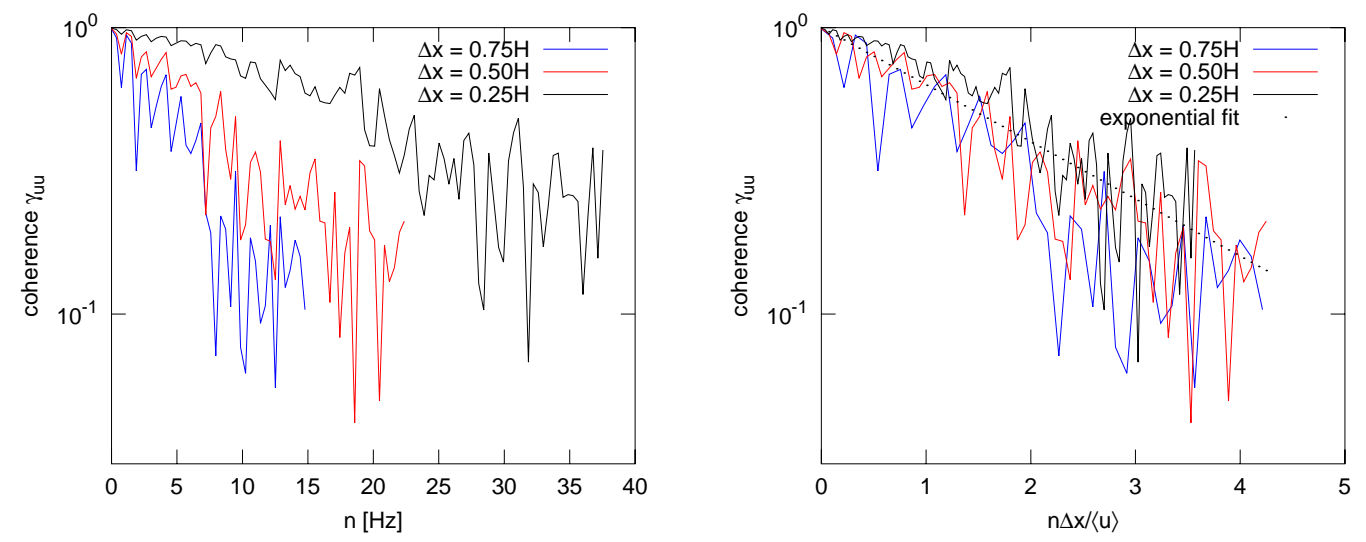

Figure 5.3: Coherence function of two points in the along-wind $x$ direction at $z / H=0.1$. $\mathrm{X}$-axis is (left) dimentional frequency; (right) nondimensional frequency $n \Delta x /\langle u\rangle$. The fitting exponent is $c_{\gamma x}=0.46 \pm 0.01$. 

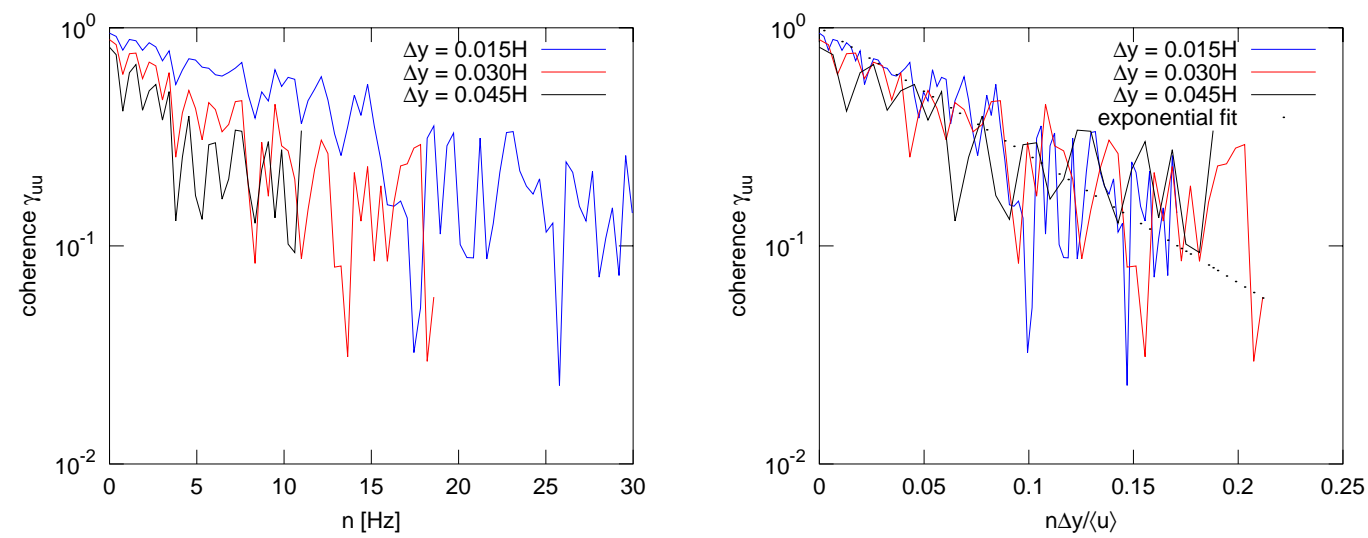

Figure 5.4: Coherence function of two points in the $y$ direction at $z / H=0.1$. X-axis is (left) dimensional frequency; (right) nondimensional frequency $n \Delta y /\langle u\rangle$. The fitting exponent is $c_{\gamma y}=13.48 \pm 0.43$.
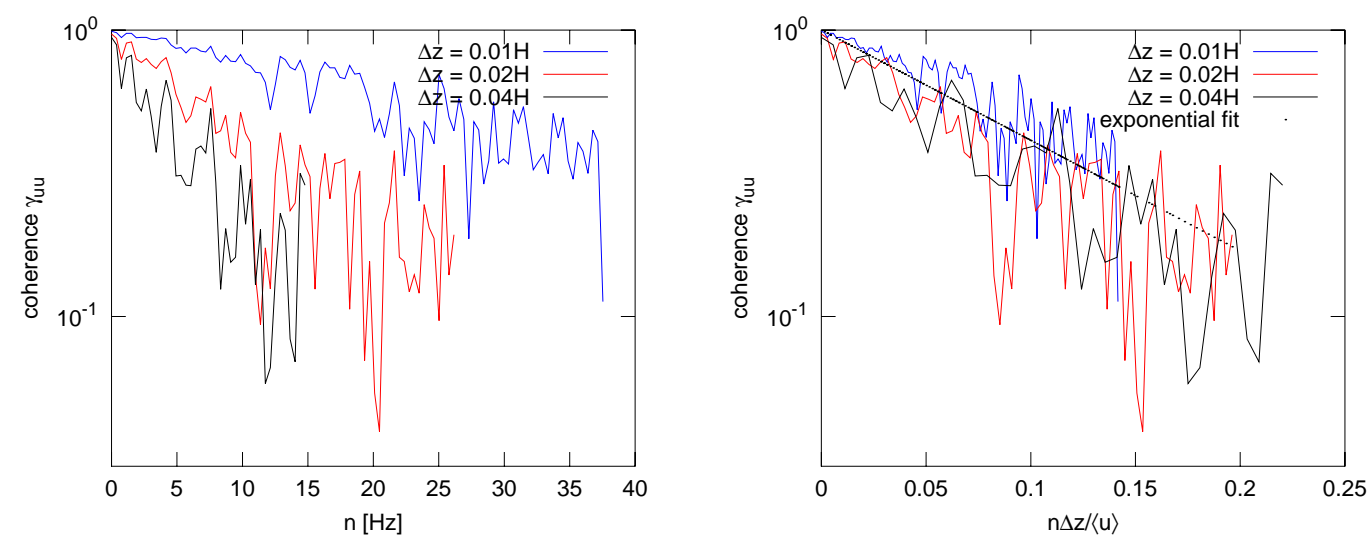

Figure 5.5: Coherence funciton of two points in the $z$ direction near $z / H=0.1$. Xaxis is (left) dimentional frequency; (right) nondimensional frequency $n \Delta z /\langle u\rangle$. Here $\langle u\rangle$ is the mean of the along-wind velocities at two points. The fitting exponent is $c_{\gamma z}=8.89 \pm 0.22$.

\section{Conclusions}

We presented a wall-modeled LES study of turbulent boundary layer flow using opensource toolbox OpenFOAM. The motivation is to use OpenFOAM in boundary layer flows with realistic flow statistics such as spectra and spatial coherence. LES simulations were performed in a rectangular parallelepiped computational domain with a uniform cubic-grid mesh. Periodic boundary conditions were applied in the horizontal directions while slip boundary condition at the top, and no-slip at the bottom for the velocity. A wall stress model was used to ensure the correct surface stresses on the wall. Initial perturbations of streaky structures were introduced to reduce the relaxation time before the fully developed turbulence. We used the solver pimpleFOAM to numerically 
integrate the filtered Navier-Stokes equations. We added an external constant pressure gradient, which is the driving force of the flow and balances the wall surface friction. one-k-equation-eddy SGS model was employed to account for the physics in unresolved scales.

Turbulence statistics were obtained for the simulated flow. We used the temporal and horizontal spatial averaging as the statistical average due to the homogeneity of the flow in these directions. The statistically averaged quantities were presented in dimensionless form. Except for the mismatch near the ground, the mean velocity and velocity gradient profiles follow reasonably well the target profiles. The second- and third-order moments of turbulence were found to be in good agreement with previous studies [3, 31]. The SGS quantities are consistent with the mathematical formulation of the SGS model. Similar to the traditional Smagorinsky model, the one-k-equationeddy model is too dissipative near the ground. The velocity components follow nearly Gaussian distributions in $y$ and $z$ direction, whereas non-Gaussian behaviors occur in $x$ direction. The power spectra are in reasonably good agreement with modified Kaimal spectra at low frequencies. At high frequencies, the spectra were found to drop much faster. Further work is planned to investigate the possibility of improving the simulation of the higher frequency spectral components.

In addition, some interesting findings from the simulations include the following:

1. The power-law representation of SGS kinetic energy and dissipation can be well explained by the equilibrium between the gradient diffusion and the dissipation in the transport equation of SGS kinetic energy. This balance is actually the basis of Smagorinsky model; Based on this balance, a model for the SGS dissipation is derived.

2. The filtered turbulent kinetic energy is approximately a logarithmic function of height;

3. The spatial coherence functions are exponential. The exponent in $x, y, z$ direction are about $0.46,13.48,8.89$, respectively. The results are consistent with the expressions commonly used in wind engineering applications and indicate the existence of the elongated streaky structures in the near-wall region.

Although a simple SGS model and a relatively coarse grid were used in the simulations, properties such as spectral density, and the spatial coherence functions of the turbulent boundary layer flow are reasonably well represented. As found in other studies, the near-ground region is still problematic. However, the knowledge of spectra and coherence is significant to the bluff body aerodynamics and structural response, which will be the topic of the future study. 


\section{References}

[1] ESDU 85020. Characteristics of atmospheric turbulence near the ground. part II: single point data for strong winds (neutral atmosphere). 1985.

[2] ESDU 86010. Characteristics of atmospheric turbulence near the ground. part III: variations in space and time for strong winds (neutral atmosphere). 1986.

[3] A. Andren, A. R. Brown, F. Graf, P. J. Mason, C. H. Moeng, and F. T. M. Nieuwstadt. Large-eddy simulation of a neutrally stratified boundary layer: A comparison of four computer codes. Q. J. R. Meteorol. Soc., 120:1457-1484, 1994.

[4] A. K. Blackadar and H. Tennekes. Asymptotic similarity in neutral barotropic planetary boundary layers. J. Atmos. Sci., 25:1015-1020, 1968.

[5] R. A. Brost, J. C. Wyngaard, and D. H. Lenschow. Marine stratocumulus layers. part II: Turbulence budgets. J. Atmos. Sci., 39:818-836, 1981.

[6] G. Chen, Q. Xiong, P. J. Morris, E. G. Paterson, A. Sergeev, and Y. Wang. Openfoam for computational fluid dynamics. Notices of the AMS, 61:354-363, 2014.

[7] S. Chen, Z. Xia, S. Pei, J. Wang, Y. Yang, Z. Xiao, and Y. Shi. Reynolds-stressconstrained large-eddy simulation of wall-bounded turbulent flows. J. Fluid Mech., 703:1-28, 2012.

[8] Matthew J. Churchfield, Ganesh Vijayakumar, James G. Brasseur, and Patrick J. Moriarty. Wind energy-related atmospheric boundary layer large-eddy simulation using OpenFOAM. In 19th Symposium on Boundary Layers and Turbulence, 2010.

[9] G. N. Coleman. Similarity statistics from a direct numerical simulation of the neutrally stratified planetary boundary layer. J. Atmos. Sci., 56:891, 1999.

[10] Eugene de Villiers. The Potential of Large Eddy Simulation for the Modeling of Wall Bounded Flows. PhD thesis, Imperial College of Science, Technology and Medicine, July 2006.

[11] J. W. Deardorff. Numerical investigation of neutral and unstable planetary boundary layers. J. Atmos. Sci., 29:91-115, 1972.

[12] M. Germano, U. Piomelli, P. Moin, and W. H. Cabot. A dynamic subgrid-scale eddy viscosity model. Phys. Fluids, A3:1760-1765, 1991.

[13] G. Grötzbach. Direct numerical and large eddy simulation of turbulent channel flows. Gulf Publishing Co., 1987.

[14] Hrvoje Jasak. Error Analysis and Estimation for the Finite Volume Method with Applications to Fluid Flows. PhD thesis, Imperial College of Science, Technology and Medicine, June 1996. 
[15] Soshi Kawai and Johan Larsson. Wall-modeling in large eddy simulation: Length scales, grid resolution, and accuracy. Phys. Fluids, 24:015105, 2012.

[16] A. G. Kravchenko and P. Moin. On the effect of numerical errors in large eddy simulations of turbulent flows. J. Comput. Phys., 131:310-322, 1997.

[17] Johan Larsson and Qiqi Wang. The prospect of using large eddy and detached eddy simulations in engineering design, and the research required to get there. Phil. Trans. R. Soc. A, 372:20130329, 2014.

[18] Jungil Lee, Minjeong Cho, and Haecheon Choi. Large eddy simulations of turbulent channel and boundary layer flows at high reynolds number with mean wall shear stress boundary condition. Phys. Fluids, 25:110808, 2013.

[19] M. Lesieur and O. Métais. New trends in large-eddy simulations of turbulence. Annu. Rev. Fluid Mech., 28:45-82, 1996.

[20] L. Lignarolo, C. Gorlé, A. Parente, and C. Benocci. Large eddy simulation of the atmospheric boundary layer using OpenFOAM. 13th International Conference on Wind Engineering, 2011.

[21] D. K. Lilly. The representation of small-scale turbulence in numerical simulation experiments. Proc. IBM Scientific Computing Symp. Environ. Sci., page 195, 1967.

[22] Charles Meneveau and Joseph Katz. Scale-invariance and turbulence models for large-eddy simulation. Annu. Rev. Fluid Mech., 32:1-32, 2000.

[23] C. H. Moeng and P. P. Sullivan. Large-eddy simulations. Encyclopedia of Atmospheric Sciences, Second Edition:232-240, 2015.

[24] Chin-Hoh Moeng and Peter P. Sullivan. A comparison of shear- and buoyancydriven planetary boundary layer flows. J. Atmos. Sci., 51:999-1022, 1994.

[25] Y. Morinishi, T. S. Lund, O. V. Vasilyev, and P. Moin. Fully conservative higher order finite difference schemes for incompressible flow. J. Comput. Phys., 143:90124, 1998.

[26] OpenFOAM. http://www.openfoam.com.

[27] OpenFOAM Foundation Ltd. OpenFOAM - The Open Source CFD Toolbox - User Guide, 2.4.0 edition, May 2015.

[28] Ugo Piomelli and Elias Balaras. Wall-layer models for large-eddy simulations. Annu. Rev. Fluid Mech., 34:349-374, 2002.

[29] Stephen B. Pope. Turbulent Flows. Cambridge University Press, August 2000.

[30] Stephen B. Pope. Ten questions concerning the large-eddy simulation of turbulent flows. New Journal of Physics, 6:1-24, 2004. 
[31] Fernando Porté-Agel, Charles Meneveau, and Marc B. Parlange. A scale-dependent dynamic model for large-eddy simulation: application to a neutral atmospheric boundary layer. J. Fluid Mech., 415:261-284, 2000.

[32] Stephen K. Robinson. Coherent motions in the turbulent boundary layer. Annu. Rev. Fluid Mech., 23:601-39, 1991.

[33] W. Schoppa and F. Hussain. Coherent structure generation in near-wall turbulence. J. Fluid Mech., 453:57-108, 2002.

[34] U. Schumann. Subgrid scale model for finite difference simulations of turbulent flows in plane channels and annuli. J. Comput. Phys., 18:376-404, 1975.

[35] Emil Simiu and Robert H. Scanlan. Wind effects on structures: An introduction to wind engineering. Wiley, 1978.

[36] Emiu Simiu, Liang Shi, and DongHun Yeo. Planetary boundary layer modeling and tall building design. Boundary-Layer Meteorol., 159:173-181, 2016.

[37] J. Smagorinsky. General circulation experiments with the primitive equations. I. the basic experiment. Mon. Weather Rev., 91:99-164, 1963.

[38] Roland B. Stull. An introduction to boundary layer meteorology. Kluwer Academic Publishers, 1988.

[39] H. Tennekes. The logarithmic wind profile. J. Atmos. Sci., 30:234-238, 1972.

[40] Peng $\mathrm{Wu}$ and Johan Meyers. A constraint for the subgrid-scale stresses in the logarithmic region of high reynolds number turbulent boundary layers: A solution to the log-layer mismatch problem. Phys. Fluids, 25:015104, 2013. 